\title{
Self-Organized Criticality and Synchronization in a Lattice Model of Integrate-and-Fire Oscillators
}

\author{
Álvaro Corral, Conrad J. Pérez, Albert Díaz-Guilera, and Alex Arenas \\ Departament de Física Fonamental, Facultat de Física, Universitat de Barcelona, Diagonal 647, E-08028 Barcelona, Spain
}

(Received 15 June 1994)

\begin{abstract}
We introduce two coupled map lattice models with nonconservative interactions and a continuous nonlinear driving. Depending on both the degree of conservation and the convexity of the driving we find different behaviors, ranging from self-organized criticality, in the sense that the distribution of events (avalanches) obeys a power law, to a macroscopic synchronization of the population of oscillators, with avalanches of the size of the system.
\end{abstract}

PACS numbers: $64.60 . \mathrm{Ht}, 05.90 .+\mathrm{m}, 87.10 .+\mathrm{e}$

A few years ago, Bak, Tang, and Wiesenfeld [1] coined the term self-organized criticality (SOC) to describe the phenomenon observed in a particular cellular automaton model, nowadays known as the sandpile model. This system is critical in analogy with classical equilibrium critical phenomena, where neither characteristic time nor length scales exist. However, in SOC one deals with dynamical nonequilibrium statistical properties, and the system evolves naturally to the critical state without any tuning of external parameters.

Several cellular automata models exhibiting SOC have been reported in the literature. In the original sandpile model of Bak, Tang, and Wiesenfeld [1], the system is perturbed externally by a random addition of sand grains. Once the slope between two contiguous cells has reached a threshold value, a fixed amount of sand is transferred to its neighbors generating a chain reaction or avalanche. The critical state is characterized by a power-law distribution of avalanche sizes, where the size is the total number of toppling events. Taking this model as a reference, different dynamical rules have been investigated leading to a wide variety of universality classes.

In the original noise driven models [1,2] it was shown that conservative interaction rules were crucial to obtain SOC [3], but more recently the interest has been focused on systems with a continuous driving such as stick-slip (SS) models of earthquakes [4-11] which exhibit SOC without a conservation law. The first of these models was studied by Feder and Feder (FF) [5]; when averaging over different samples a power-law distribution of avalanche sizes is observed, but simple realizations exhibit a periodical behavior that depends on the initial configuration. Furthermore, the role played by nonconservation is unclear in terms of the redistribution of forces after a slip process. Lately, however, it was shown that deterministically driven models with other interaction rules do exhibit SOC for different levels of conservation $[6,7,9,11]$.

All these SS models have strong analogies with certain models of integrate-and-fire oscillators (IFO), widely analyzed to study the behavior of populations of pace- maker cells and other biological systems [12-19]. Of particular interest to us is the resemblance with a model discussed by Mirollo and Strogatz (MS) [17], who considered a large assembly of IFO to show that under certain conditions the stationary state presents perfect synchrony among all the elements of the population. These conditions are (i) a nonlinear convex driving for the individual dynamics of each unit and (ii) long-range interactions between them. With a linear driving it is possible to have some temporal coherence-entrainment-but not to ensure that the whole assembly will be synchronized. This study highlights the role of the driving mechanism on the final state of the system. In the SS and MS models there is an intrinsic dynamics - the driving - leading the elements of the system to the threshold. When a block (oscillator) reaches the threshold it slips (fires), and this produces a change in the state of its neighbors. This process can produce further slips (firings) generating an avalanche. During the propagation of an avalanche, the natural dynamics is stopped and the collective behavior is determined by some interaction rules. When the avalanche is completed, all the elements are below the threshold and the driving governs again the dynamics of the system. Notice that while in SOC models one studies the avalanche size distribution, in a model of IFO one describes the state of the system after the end of the interactive process, looking, for instance, at the level of mutual entrainment between units. Essentially, both models contain the same basic ingredients, and from this point of view SOC and synchronization might be considered as two sides of the same coin.

In view of the analogy between models displaying SOC and IFO models showing complex patterns of synchronization, the purpose of this Letter is to develop a general framework where a diversity of macroscopic behaviors can be observed by an appropriate choice of the parameters that describe the dynamics of the elementary units as well as the interaction rules among them. Keeping this goal in mind, we are going to analyze the effect of a nonlinear convex driving in models that 
do display SOC with a linear driving $[6,20]$ and observe under which conditions SOC breaks down and relaxation oscillations show up.

We study a coupled map model on a two-dimensional square lattice of size $L \times L$ with open boundary conditions. Each cell is characterized by two variables: a phase variable $\varphi$ that increases linearly with time, and a state variable $E$, which we call energy but can have different physical interpretations. They evolve in time as [12]

$$
\begin{gathered}
d \varphi / d t=1, \\
d E / d t=\gamma(K-E),
\end{gathered}
$$

with $K=1 /\left(1-e^{-\gamma}\right)$. Assuming $E(\varphi=0)=0$ and $E(\varphi=1)=1$, these variables are related through the following relations [17]:

$$
\begin{gathered}
E(\varphi)=K\left[1-e^{-\gamma \varphi}\right], \\
\varphi(E)=\frac{1}{\gamma} \ln \frac{K}{K-E} .
\end{gathered}
$$

The cycle period has been taken to be one without loss of generality, and $\gamma$ is a measure of the convexity of the driving. From Eqs. (1) we can see that only when $\gamma=0$ (linear driving) the energy of the cells will increase uniformly with time.

Once a cell becomes critical $\left(E_{i, j} \geq E_{c}=1\right)$ it "fires" and transfers energy to the four nearest neighboring cells according to the following rules $[2,6]$ :

$$
\begin{aligned}
E_{i \pm 1, j} & \rightarrow E_{i \pm 1, j}+\epsilon E_{i, j}, \\
E_{i, j \pm 1} & \rightarrow E_{i, j \pm 1}+\epsilon E_{i, j}, \\
E_{i, j} & \rightarrow 0,
\end{aligned}
$$

where $\epsilon$ is the dissipative rate and $\epsilon=0.25$ corresponds to a conservative dynamics. This model differs from FF and MS where the interaction rules are

$$
\begin{aligned}
E_{i \pm 1, j} & \rightarrow E_{i \pm 1, j}+\epsilon, \\
E_{i, j \pm 1} & \rightarrow E_{i, j \pm 1}+\epsilon, \\
E_{i, j} & \rightarrow 0 .
\end{aligned}
$$

In this case the system is not conservative for any value of $\epsilon$. These rules are a short-range version of the infiniteranged rules used in Ref. [17]. Notice that the concept of absorption is removed from the MS model, so that when a cell becomes critical either by its own dynamics or by the interactive process, it always transfers energy to (modifies the phase of) its neighbors. The energy of a cell may be larger than $E_{c}=1$, but this property is not inconsistent with Eqs. (2) since a given site may only have $E>1$ when an avalanche is triggered, i.e., when the interaction rules control the dynamic behavior of the system. However, there is an important difference between the two interaction rules: In terms of IFO (3a) implies that the phase response of an oscillator that receives a pulse not only depends on the current phase but also on the energy of the element that has fired, while for (3b) the energy of the firing cell is irrelevant.
Starting with a random distribution of phases, we let the phase of the cells evolve according to Eqs. (1), until one of them reaches the threshold value and energy is redistributed following (3). We have introduced a certain amount of noise to ensure that the driving makes only one cell to fire. If several cells reach the threshold simultaneously the noise discriminates between them by choosing one at random. In this way it is ensured that two avalanches cannot overlap. Once all cells have an energy below the threshold, the system is driven again. This dynamics involves two time scales, one for the intrinsic dynamics of the units and another for the interactions; in SS models of earthquakes the first scale corresponds to the motion of the tectonic plates and the second one to the duration of the earthquakes; since the former is orders of magnitude larger than the second one, we are going to assume that the avalanches are instantaneous. This assumption has also been made in recent studies on IFO $[17,21]$, but in order to discuss more realistic models it is necessary to take into account some further ingredients, such as the time that signals need to propagate through the lattice or the refractory time associated with the response of a cell, just to mention a few. Their effects are currently under study.

We have performed numerical simulations for different values of $\epsilon$ and $\gamma$ on lattice sizes up to $L=64$. In Fig. 1 we have plotted the schematic phase diagram of

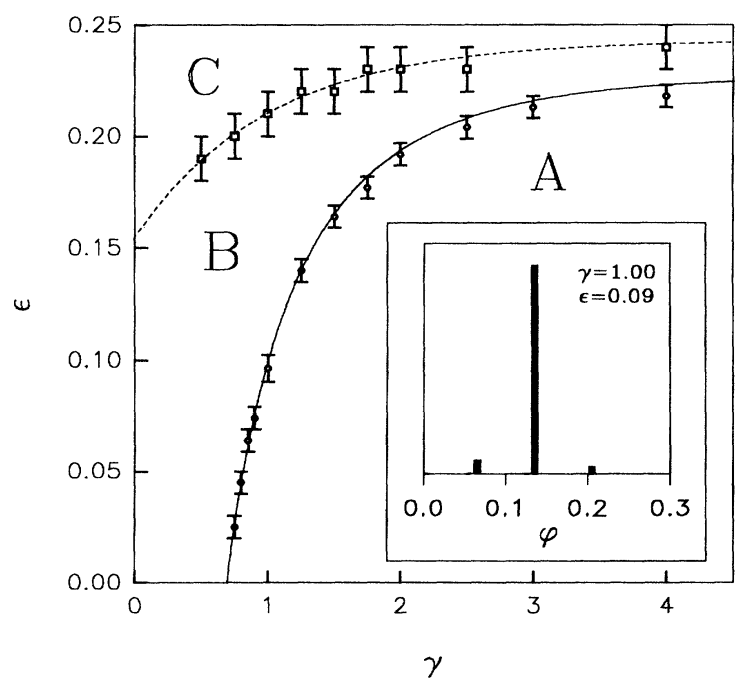

FIG. 1. Schematic phase diagram in terms of $\gamma$ (the convexity of the driving) and $\epsilon$ (the level of conservation) for model (3a). The symbols correspond to the phase transitions observed in the simulations. For the B-C transition the error bars denote that above them we have always found SOC while below them there is no power-law behavior. For the A-B transition the error bars are given by the standard deviation over ten measures. The solid line corresponds to our analytical result (7) and the dashed line is an exponential fit to the numerical data. The inset displays the distribution of phases after an avalanche in region A for a system of size $L=64$ with $\gamma=1.00$ and $\epsilon=0.09$. 
model (3a) in terms of these parameters. Three regions with a clear different macroscopic behavior after $3 \times 10^{6}$ avalanches are observed. The features of each region are discussed in the next paragraphs.

In region $\mathrm{A}$ there is only one type of avalanche that sweeps the whole system when it has reached the stationary state. The avalanche starts at a given cell and propagates forming a diamond-shaped front of firing cells due to the underlying square lattice structure. Each site fires exactly once, and when the avalanche is over the driving acts until a cell fires again. This cell (seed) is always the same. Based on the structure of the front we can describe analytically this repetitive situation. Once the seed has fired it gets four firings from its neighbors and does not fire again. Its nearest neighbors get one firing from the seed, then fire, and then receive three firings from their neighbors. In this way, all the cells get a fixed number of firings (before and after reaching the threshold) which only depends on their position on the lattice. Once the avalanche is over, the system has a well-defined distribution of energies which depends on the interaction rules: for ( $3 b)$ the seed is at $4 \epsilon$, the cells which form the vertices of the front are at $3 \epsilon$, the boundaries are at $\epsilon$, the corners have zero energy, and the remaining cells (the bulk) are at $2 \epsilon$. Now, all the cells increase their respective phases uniformly, the seed being the one that reaches the threshold first. The avalanche must be able to reach the zero energy cells on the corners and enable them to fire. Thus the energy corresponding to a phase increment of $1-\varphi(4 \epsilon)$ plus the energy of two firings coming from their neighbors must be larger than the threshold, i.e.,

$$
E[1-\varphi(4 \epsilon)]+2 \epsilon \geq 1 .
$$

A simple calculation yields

$$
\epsilon \leq \frac{2-K}{4}
$$

which is the relation between $\epsilon$ and $\gamma$ that must be satisfied for model ( $3 b$ ) in order to show relaxation oscillations involving all the cells. This relation has been checked through simulations finding an excellent agreement. Notice that after each avalanche the cells forming the bulk have the same phase. Therefore, the system presents a macroscopic synchronization among almost all the elements of the lattice.

Now, let us consider rule (3a) which gives SOC for a linear driving. The main difference with respect to the above situation is that we have to replace $\epsilon$ by an effective value $\bar{\epsilon}$, since the energy of a given site can be larger than 1 when the avalanche propagates through the lattice. Within a mean-field approximation we will assume that this energy is the same for each cell. For this model, when the avalanche has finished, the seed has a phase $\varphi(4 \bar{\epsilon})$, and to fire again it has to increment it by an amount $1-\varphi(4 \bar{\epsilon})$. Since its neighbors are at $\varphi(3 \bar{\epsilon})$, the condition to repeat permanently this situation is

$$
4 \bar{\epsilon}=4 \epsilon\{E[\varphi(3 \bar{\epsilon})+1-\varphi(4 \bar{\epsilon})]+\epsilon\} .
$$

This equality, in addition to Eq. (5) (replacing $\epsilon$ by $\bar{\epsilon}$ ), gives the following relationship between $\epsilon$ and $\gamma$ :

$$
\epsilon \leq \frac{1}{16}\left[\sqrt{K^{2}-52 K+164}-(K+6)\right] .
$$

When this condition is fulfilled, only relaxation oscillations of the size of the system can survive in the stationary state. The curve corresponding to the equality has been plotted in Fig. 1 (solid line) and has been corroborated through simulations (circles). Each symbol is an average over ten different random initial distributions of phases. The inset in Fig. 1 is an example of the histogram of phases after an avalanche. The height of the peak at $\varphi(2 \bar{\epsilon})$ scales as $L^{2}$, the other two at $\varphi(\bar{\epsilon})$ and at $\varphi(3 \bar{\epsilon})$ scale as $L$, and finally there is a finite number of cells that have zero phase and $\varphi(4 \bar{\epsilon})$ whose height is negligible in this plot.

Thus we have a coupled map lattice model that exhibits SOC when it is driven linearly and relaxation oscillations when the nonlinear driving is sufficiently important. Now we focus on the intermediate behavior (region $B$ in Fig. 1). Starting in the region with a macroscopic degree of synchronization we fix $\gamma$ and increase $\epsilon$. Slightly above the curve given by Eq. (7) an avalanche sweeping all of the lattice cannot repeat any more since the next one will be unable to reach some of the cells in the boundaries, and these cells will be the starting point of future avalanches. This fact gives rise to a periodic behavior with a discrete distribution of a few avalanche sizes. This is indeed what we have observed in the simulations; only some values of avalanche sizes are present and those are very sensitive to the initial random configuration. By increasing $\epsilon$ the distribution of avalanche sizes, $D(s)$, varies from the discrete distribution to a continuous one with some characteristic lengths which can be identified by the peaks in Fig. 2; the peaks in $D(s)$ scale with different powers of $L$ and a finite-size scaling is not possible in this situation. Furthermore, this stationary distribution does not depend on the initial conditions.

For larger values of $\epsilon$ (region $C$ of Fig. 1), close to the conservation line, the peaks disappear and we get a power-law decay with an exponential cutoff. In this case the distribution $D(s)$, for different values of $L$, can be fitted into a single curve by a proper finite-size scaling (see Fig. 3), which is the hallmark of SOC. However, we have to stress that for large values of $\gamma$ the power-law behavior remains, but it is followed by a peak before the exponential cutoff. Nevertheless, the whole distribution of avalanche sizes still presents a good finite-size scaling. Another point to remark is that the exponents of the finite-size scaling ( $\alpha$ and $\beta$ ), and hence the slope of $D(s)$ on a $\log -\log$ scale, are continuous functions of $\gamma$ and $\epsilon$; thus we can conclude that the nonlinear driving gives rise to a broader spectrum of exponents than that 


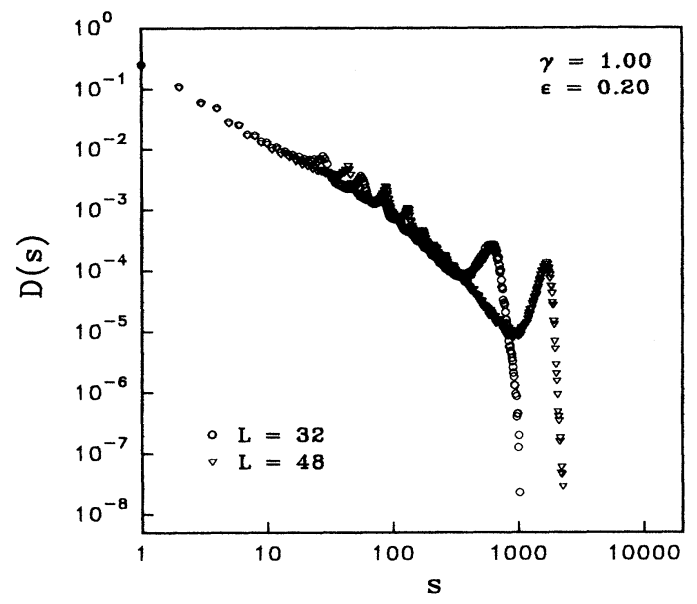

FIG. 2. Distribution of avalanche sizes in region B.

studied in the linear case [6]. We have identified the transition from regions $\mathrm{B}$ to $\mathrm{C}$ (squares in Fig. 1) by fixing $\gamma$ and increasing $\epsilon$ up to the appearance of a finitesize scaling. The dashed line is an exponential fit of the numerical results that we extrapolate to the linear driving case $(\gamma=0)$ and for large values of the convexity of the driving. The extrapolation to the linear driving gives a value of $\epsilon \simeq 0.16$ below which there would be no SOC, which is in agreement with recent results by Grassberger [11] on very large lattices.

In summary, we have studied two coupled map lattice models where different behaviors, ranging from a power law decay of the distribution of avalanche sizes and finite-size scaling, characteristics of SOC, to relaxation oscillations with a macroscopic degree of synchronization, can be observed. This is achieved by an appropriate

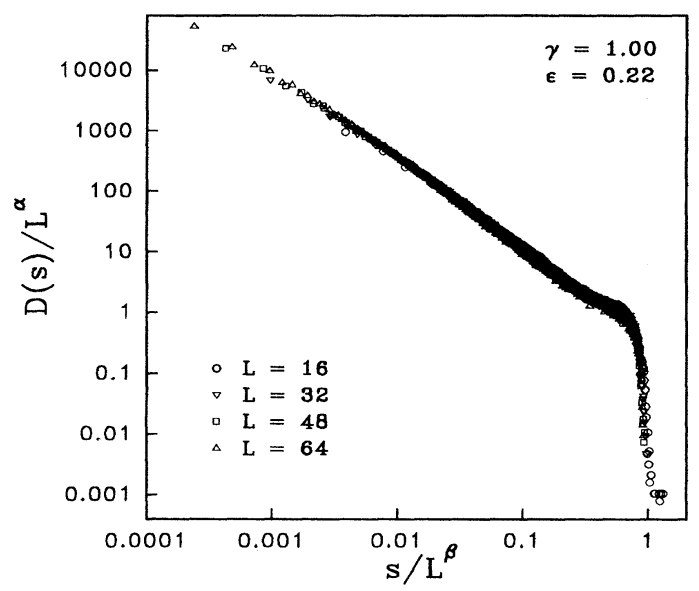

FIG. 3. Finite-size scaling ansatz of the distribution of avalanche sizes in region $C$. In this case $\alpha=-2.9 \pm 0.1$ and $\beta=2.0 \pm 0.1$. choice of the parameters governing the dynamics of the elementary units and the interaction rules between cells. Based on the spatial structure that an avalanche sweeping the whole lattice has, we have found an analytical relation between the convexity of the driving and the level of conservation in the interaction rules that ensures that such an avalanche will repeat continuously. This is the condition that gives rise to relaxation oscillations.

The authors are indebted to K. Christensen for very fruitful discussions and for a critical reading of the manuscript. This work has been supported by CICyT of the Spanish Government, Grant No. PB92-0863.

[1] P. Bak, C. Tang, and K. Wiesenfeld, Phys. Rev. A 38, 364 (1988).

[2] Y.-C. Zhang, Phys. Rev. Lett. 63, 470 (1989).

[3] S. S. Manna, L. B. Kiss, and J. Kertesz, J. Stat. Phys. 22, 923 (1990).

[4] R. Burridge and L. Knopoff, Bull. Seismol. Soc. Am. 57, 341 (1967).

[5] H. J.S. Feder and J. Feder, Phys. Rev. Lett. 66, 2669 (1991).

[6] Z. Olami, H. J.S. Feder, and K. Christensen, Phys. Rev. Lett. 68, 1244 (1992); K. Christensen and Z. Olami, Phys. Rev. A 46, 1829 (1992).

[7] K. Christensen, Z. Olami, and P. Bak, Phys. Rev. Lett. 68, 2417 (1992).

[8] Z. Olami and K. Christensen, Phys. Rev. A 46, R1720 (1992).

[9] J.E. S. Socolar, G. Grinstein, and C. Jayaprakash, Phys. Rev. E 47, 2366 (1993).

[10] J. B. Rundle and W. Klein, J. Stat. Phys. 72, 405 (1993).

[11] P. Grassberger, Phys. Rev. E 49, 2436 (1994).

[12] C. S. Peskin, Mathematical Aspects of Heart Physiology (Courant Institute of Mathematical Sciences, New York University, New York, 1975).

[13] A.T. Winfree, The Geometry of Biological Time (Springer-Verlag, New York, 1980).

[14] G. B. Ermentrout, J. Math. Biol. 22, 1 (1985).

[15] A. Sherman, J. Rinzel, and J. Keizer, Biophys. J. 54, 411 (1988); R. D. Traub, R. Miles, and R. K. S. Wong, Science 243, 1319 (1989).

[16] L. F. Abbott, J. Phys. A 23, 3835 (1990).

[17] R. E. Mirollo and S. H. Strogatz, SIAM J. Appl. Math. 50, 1645 (1990).

[18] Y. Kuramoto, Physica (Amsterdam) 50D, 15 (1991).

[19] J. J. Hopfield, Phys. Today 47, 40 (1994); S. H. Strogatz and I. Stewart, Sci. Am. 269 (No. 6), 68 (1993).

[20] It is important to remark that in our case the nonlinearity is introduced in the driving whereas in Ref. [9] the system is driven uniformly and the nonlinearity is introduced in the interaction rules.

[21] C. Vanvreeswijk and L. F. Abbott, SIAM J. Appl. Math. 53, 253 (1993). 